RAIRO-Inf. Theor. Appl. 41 (2007) 45-56

DOI: $10.1051 /$ ita:2007006

\title{
REAL AND COMPLEX PSEUDOZERO SETS FOR POLYNOMIALS WITH APPLICATIONS
}

\author{
Stef Graillat ${ }^{1}$ and Philippe Langlois ${ }^{2}$
}

\begin{abstract}
Pseudozeros are useful to describe how perturbations of polynomial coefficients affect its zeros. We compare two types of pseudozero sets: the complex and the real pseudozero sets. These sets differ with respect to the type of perturbations. The first set - complex perturbations of a complex polynomial - has been intensively studied while the second one - real perturbations of a real polynomial - seems to have received little attention. We present a computable formula for the real pseudozero set and a comparison between these two pseudozero sets. We conclude that the complex pseudozero sets have to be preferred except when the perturbed real polynomials admit non-real zeros. We also give some applications of pseudozero set in control theory.
\end{abstract}

Mathematics Subject Classification. 65F35, 68W30.

\section{INTRODUCTION}

The computation of polynomial roots is commonly used in numerous applications in scientific computing or engineering. In practice, the real or complex polynomial coefficients are often approximate values. Two well known sources of such approximation are data uncertainties and rounding errors. The sensitivity of the roots with respect to these perturbations of the polynomial coefficients has been studied with several kinds of methods.

Analytical sensitivity analysis introduces a condition number that bounds the magnitudes of the (first order) changes of the roots with respect to the coefficient perturbations. Numerous results in this direction are available, see for example

\footnotetext{
Keywords and phrases. Polynomial root, pseudozero set, uncertainty, perturbation, stability.

${ }^{1}$ Laboratoire LIP6, département Calcul scientifique, université Pierre et Marie Curie, 4 place Jussieu, F-75252, Paris Cedex 05, France; stef.graillat@lip6.fr

2 DALI Project, Laboratory LP2A, université de Perpignan, 52, avenue Paul Alduy, 66860 Perpignan Cedex, France; langlois@univ-perp.fr
}

(c) EDP Sciences 2007 
Gautschi [2] or Wilkinson [16]. Representing coefficient uncertainty with intervals and computing with interval arithmetic yield over-sets that enclose (sometimes pessimistically) the perturbed roots [7]. When the coefficient uncertainty is assumed to satisfy a given probabilistic behavior, stochastic arithmetic of Vignes and Chesneaux and its associated library CADNA provide a probabilistic estimate of perturbed roots $[1,15]$. Continuous sensitivity analysis, introduced by Ostrowski [11], considers the uncertainty of the coefficients as a continuity problem. The most powerful tool of this last type of methods seems to be the pseudozero set of a polynomial we focus hereafter. Roughly speaking, it is the set of the roots of polynomials being near to a given polynomial. This set was first introduced by Mosier [10]. A very recent survey about pseudozero set is proposed by Stetter [13].

In this paper, we study two kinds of pseudozero sets. The first one is the complex pseudozero set: it is the set of complex numbers that are the roots of polynomials with complex coefficients being near to a given polynomial $p$ with complex coefficients. The second one is the real pseudozero set, that is the set of complex numbers that are the roots of polynomials with real coefficients being near to a given polynomial $p$ with real coefficients.

For a given polynomial with real coefficients, it makes sense to compute both complex and real pseudozero sets even if the latter may be closer to the physical problem the polynomial represent. This is the case when the polynomial coefficients describe non-complex physical values as for example in transfer function for control theory. Previous works of the authors illustrate how the complex pseudozero set solves stability problems in this area [3,4]. Another motivation to constrain the pseudozero set to real perturbations comes from finite precision computation since the rounding error in real coefficients represented by fixed or floating numbers is always a non-complex perturbation. The aim of this paper is to compare these two pseudozero sets and evaluate which one is the more convenient and the easiest to compute.

Up to our knowledge, published results often consider only complex pseudozero sets. The real pseudozero set has not been studied a lot before. Hereafter we provide a computable formula for the real pseudozero set and we justify that the complex pseudozero set is more convenient than the real one.

The paper is organized as follows. In Section 2, we recall some definitions and known results about the complex pseudozero set. In Section 3, we introduce the real pseudozero set and we propose a computable expression for this set. In Section 4, we present some numerical simulations and we compare the real and complex pseudozero sets. The Section 5 is devoted to applications of pseudozero set in control theory and to robustness problems in particular.

\section{Complex Pseudozero Set of polynomials}

Let $\mathcal{P}_{n}(\mathbb{C})$ be the linear space of polynomials of degree at most $n$ with complex coefficients $(n \geq 1)$. Let $p$ be a polynomial of $\mathcal{P}_{n}(\mathbb{C})$ with complex coefficients $p_{i}$ 
$(0 \leq i \leq n)$ such that

$$
p(z)=\sum_{i=0}^{n} p_{i} z^{i}
$$

Representing $p$ by the vector $\left(p_{0}, p_{1}, \ldots, p_{n}\right)^{T}$ of its coefficients, we identify the norm on $\mathcal{P}_{n}(\mathbb{C})$ to the 2 -norm of the corresponding vector in $\mathbb{C}^{n+1}$. Throughout this paper, we will only consider this 2 -norm we denote $\|\cdot\|$. It means that

$$
\|p\|=\left(\sum_{i=0}^{n}\left|p_{i}\right|^{2}\right)^{1 / 2}
$$

The following real parameter $\varepsilon$ bounds the uncertainty of the coefficients of $p$. Given such an $\varepsilon>0$, a complex $\varepsilon$-neighborhood of $p$ is the set of all polynomials of $\mathcal{P}_{n}(\mathbb{C})$, close enough to $p$, that is,

$$
N_{\varepsilon}(p)=\left\{\widehat{p} \in \mathcal{P}_{n}(\mathbb{C}):\|p-\widehat{p}\| \leq \varepsilon\right\}
$$

The complex $\varepsilon$-pseudozero set of $p$ is defined to include all the zeros of the $\varepsilon$-neighborhood of $p$. A definition of this set is

$$
Z_{\varepsilon}(p)=\left\{z \in \mathbb{C}: \widehat{p}(z)=0 \text { for some } \widehat{p} \in N_{\varepsilon}(p)\right\}
$$

Theorem 2.1 below provides a computable counterpart of this definition.

Theorem 2.1 (Trefethen and Toh [14]). The complex $\varepsilon$-pseudozero set of p verifies

$$
Z_{\varepsilon}(p)=\left\{z \in \mathbb{C}: g(z):=\frac{|p(z)|}{\|\underline{z}\|} \leq \varepsilon\right\},
$$

where $\underline{z}=\left(1, z, \ldots, z^{n}\right)^{T}$

This theorem was proved in [14] for the 2-norm and in [12] for an arbitrary norm. We recall the proof of [14].

Proof. If $z \in Z_{\varepsilon}(p)$ then there exists $\widehat{p} \in \mathcal{P}_{n}(\mathbb{C})$ such that $\widehat{p}(z)=0$ and $\|p-\widehat{p}\| \leq \varepsilon$. From Hölder's inequality $\left|x^{T} y\right| \leq\|x\|\|y\|$, we get

$$
|p(z)|=|p(z)-\widehat{p}(z)|=\left|\sum_{i=0}^{n}\left(p_{i}-\widehat{p}_{i}\right) z^{i}\right| \leq\|p-\widehat{p}\|\|\underline{z}\| .
$$

It follows that $|p(z)| \leq \varepsilon\|\underline{z}\|$.

Conversely, let $u \in \mathbb{C}$ be such that $|p(u)| \leq \varepsilon\|\underline{u}\|$. If $u \neq 0$, we can write $u=|u| e^{i \theta}, \theta \in[0,2 \pi)$ with $|u|>0$. Let us introduce the polynomials $r$ and $p_{u}$ 
defined by

$$
\begin{aligned}
r(z) & =\sum_{k=0}^{n} r_{k} z^{k}, \text { with } \quad r_{k}=|u|^{k} e^{-i k \theta}, \text { and } \\
p_{u}(z) & =p(z)-\frac{p(u)}{r(u)} r(z) .
\end{aligned}
$$

It is clear that $r(u)=\|\underline{u}\|^{2}=\|r\|^{2}$, and $p_{u}(u)=0$. So we have

$$
\left\|p-p_{u}\right\|=\frac{|p(u)|}{|r(u)|}\|r\| \leq \varepsilon .
$$

Hence we obtain that $u$ belongs to $Z_{\varepsilon}(p)$.

If $u=0$, let us define $p_{u}(z)=p(z)-p(u)$. It is clear that $p_{u}(u)=0$. Besides, we have $\left\|p-p_{u}\right\|=|p(u)| \leq \varepsilon$ by hypothesis. In the same way, we get that $u$ belongs to $Z_{\varepsilon}(p)$.

This theorem gives us an efficient way to compute the complex pseudozero set. The $\varepsilon$-pseudozeros of $p$ belong to the interior of the area defined by the contour level (of value $\varepsilon$ ) of the normalized residual $|p(z)| /\|z\|$. Using for example MATLAB primitives, following Algorithm 1 easily plots the $\varepsilon$-pseudozeros of a polynomial $p$ for a given coefficient uncertainty $\varepsilon$.

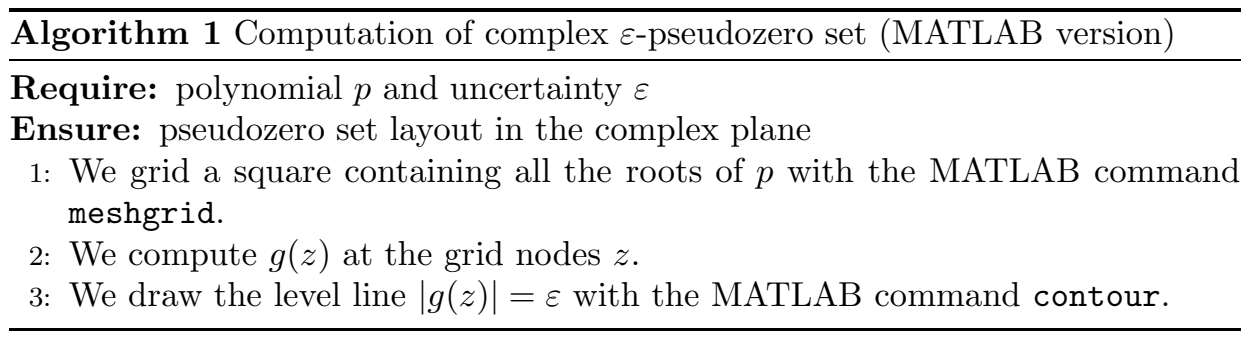

\section{ReAl PSEUdozero SET OF POLYNOMIALS}

Now, we introduce the real pseudozero set and a computable expression for this set.

The notations are similar to the complex case. For $n \geq 1$, let $\mathcal{P}_{n}(\mathbb{R})$ be the linear space of polynomials of at most degree $n$ with real coefficients. A polynomial $p$ in $\mathcal{P}_{n}(\mathbb{R})$ is represented by the vector $\left(p_{0}, p_{1}, \ldots, p_{n}\right)^{T}$ of its coefficients, so $p(z)=\sum_{i=0}^{n} p_{i} z^{i}$. Again we identify the norm $\|\cdot\|$ on $\mathcal{P}_{n}(\mathbb{R})$ to the 2-norm on $\mathbb{R}^{n+1}$ of the corresponding vector of its coefficients. Relation (1) is still valid to define this norm. 
Let $\varepsilon$ be a given bound of the polynomial coefficient uncertainties. A real $\varepsilon$-neighborhood of $p$ is the set of all polynomials of $\mathcal{P}_{n}(\mathbb{R})$, close enough to $p$, that is,

$$
N_{\varepsilon}^{R}(p)=\left\{\widehat{p} \in \mathcal{P}_{n}(\mathbb{R}):\|p-\widehat{p}\| \leq \varepsilon\right\} .
$$

Then the real $\varepsilon$-pseudozero set of $p$ is defined to include all the zeros of the real $\varepsilon$-neighborhood of $p$. A definition of this set is

$$
Z_{\varepsilon}^{R}(p)=\left\{z \in \mathbb{C}: \widehat{p}(z)=0 \text { for some } \widehat{p} \in N_{\varepsilon}^{R}(p)\right\} .
$$

For $\varepsilon=0$, the pseudozero set $Z_{0}^{R}(p)$ is the set of the roots of $p$ we denote $Z(p)$.

One can easily notice that the real $\varepsilon$-pseudozero set $Z_{\varepsilon}^{R}(p)$ is symmetric with respect to the real axis.

Proposition 3.1. The real $\varepsilon$-pseudozero set $Z_{\varepsilon}^{R}(p)$ is symmetric with respect to the real axis.

Proof. Let $z \in Z_{\varepsilon}^{R}(p)$. It means that there exists $q \in N_{\varepsilon}^{R}(p)$ such that $q(z)=0$. As the polynomial $q$ has real coefficients, this implies that $q(\bar{z})=0$. So $\bar{z} \in Z_{\varepsilon}^{R}(p)$.

Following Theorem 3.2 provides a computable counterpart of this definition. It can be considered as a special case of spectral value sets for the companion matrix using structured perturbations [5] when the polynomial is monic. We prove it hereafter using arguments developed in [6] but staying in the field of polynomials. The proof also deals with non-monic polynomials. We define for $x, y \in \mathbb{R}^{n+1}$,

$$
d(x, \mathbb{R} y)=\inf _{\alpha \in \mathbb{R}}\|x-\alpha y\|,
$$

the distance of a point $x \in \mathbb{R}^{n+1}$ from the linear subspace $\mathbb{R} y=\{\alpha y, \alpha \in \mathbb{R}\}$.

Theorem 3.2. The real $\varepsilon$-pseudozero set of $p$ verifies

$$
Z_{\varepsilon}^{R}(p)=Z(p) \cup\left\{z \in \mathbb{C} \backslash Z(p): h(z):=d\left(G_{R}(z), \mathbb{R} G_{I}(z)\right) \geq \frac{1}{\varepsilon}\right\},
$$

where $G_{R}(z)$ and $G_{I}(z)$ are the real and imaginary parts of

$$
G(z)=\frac{1}{p(z)}\left(1, z, \ldots, z^{n}\right)^{T}, z \in \mathbb{C} \backslash Z(p) .
$$

Proof. Let $z \in Z_{\varepsilon}^{R}(p)$. If $p(z)=0$ then $z \in Z(p)$ else there exists $q \in N_{\varepsilon}^{R}(p)$ such that $q(z)=0$. In this case, we have $p(z)=p(z)-q(z)=(p-q)^{T} \underline{z}$, where $\underline{z}=\left(1, z, \ldots, z^{n}\right)^{T}$. It follows that $1=(p-q)^{T} G(z)$. Hence we have $1=(p-q)^{T} G_{R}(u)+i(p-q)^{T} G_{I}(u)$ and so

$$
\left\{\begin{array}{l}
(p-q)^{T} G_{R}(u)=1 \\
(p-q)^{T} G_{I}(u)=0
\end{array}\right.
$$


As a consequence, we have $\|p-q\|\left\|G_{R}(u)-\alpha G_{I}(u)\right\| \geq 1$, for all $\alpha \in \mathbb{R}$. We conclude that

$$
d\left(G_{R}(u), \mathbb{R} G_{I}(u)\right) \geq \frac{1}{\|p-q\|} \geq \frac{1}{\varepsilon} .
$$

Conversely, let $z \in Z(p) \cup\left\{z \in \mathbb{C} \backslash Z(p): d\left(G_{R}(z), \mathbb{R} G_{I}(z)\right) \geq \frac{1}{\varepsilon}\right\}$. If $z$ belongs to $Z(p)$ then it belongs to $Z_{\varepsilon}^{R}(p)$. Otherwise $z$ satisfies $\|G(z)\| \geq 1 / \varepsilon$. From a duality theorem (see [9], p. 119), there exists a vector $u \in \mathbb{R}^{n+1}$ with $\|u\|=1$ satisfying

$$
u^{T} G_{R}(z)=d\left(G_{R}(z), \mathbb{R} G_{I}(z)\right) \quad \text { and } \quad u^{T} G_{I}(z)=0
$$

Let us consider the real polynomial

$$
q=p-\frac{u}{d\left(G_{R}(z), \mathbb{R} G_{I}(z)\right)}
$$

We have

$$
q(z)=p(z)-\frac{u^{T} \underline{z}}{d\left(G_{R}(z), \mathbb{R} G_{I}(z)\right)}=p(z)-\frac{p(z) u^{T} G(z)}{d\left(G_{R}(z), \mathbb{R} G_{I}(z)\right)}=0 .
$$

Furthermore we have $\|q-p\|=1 / d\left(G_{R}(z), \mathbb{R} G_{I}(z)\right)$. It follows $\|p-q\| \leq \varepsilon$.

To compute the real $\varepsilon$-pseudozero set $Z_{\varepsilon}^{R}(p)$, we only have to evaluate the distance $d\left(G_{R}(z), \mathbb{R} G_{I}(z)\right)$. We recall that $\|\cdot\|$ is the 2 -norm and we denote with $\langle\cdot, \cdot\rangle$ the associated inner product. In this case we have

$$
d(x, \mathbb{R} y)= \begin{cases}\sqrt{\|x\|^{2}-\frac{\langle x, y\rangle^{2}}{\|y\|^{2}}} & \text { if } y \neq 0 \\ \|x\| & \text { if } y=0\end{cases}
$$

Then the real pseudozero set is also the interior of the area defined by the level contour of function $h$ defined by Relation 4 . We can compute the real $\varepsilon$-pseudozero set with following Algorithm 2.

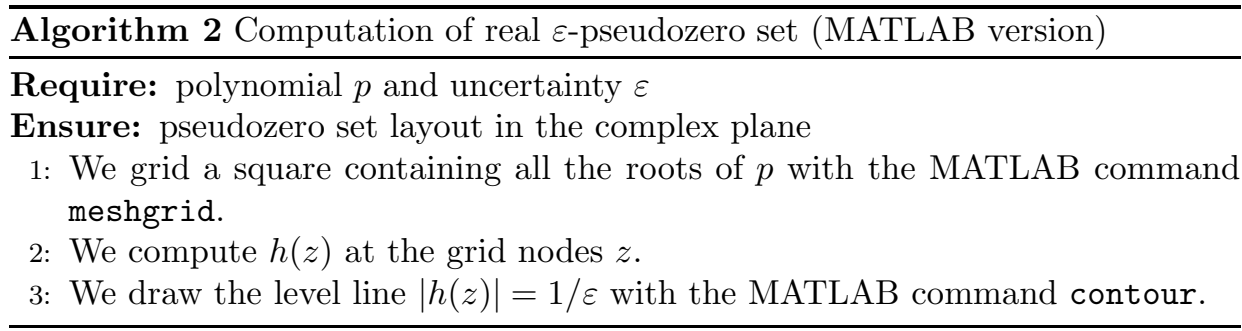




\section{NUMERICAL SIMULATIONS AND COMPARISONS}

In this section, we present some numerical simulations and we compare the real and complex pseudozero sets. This comparison is motivated since it is more difficult to compute the real pseudozero set than the complex pseudozero set. It is clear that we always have $Z_{\varepsilon}^{R}(p) \subset Z_{\varepsilon}(p)$.

Proposition 4.1. Let $p$ be a polynomial of $\mathcal{P}_{n}(\mathbb{R})$. Then the real pseudozero set is included in the complex pseudozero set, i.e.,

$$
Z_{\varepsilon}^{R}(p) \subset Z_{\varepsilon}(p) .
$$

Proof. Let $z$ belonging to $Z_{\varepsilon}^{R}(p)$. It means that there exists a polynomial $q$ in $\mathcal{P}_{n}(\mathbb{R})$ such that $q(z)=0$. Since $\mathcal{P}_{n}(\mathbb{R})$ is included in $\mathcal{P}_{n}(\mathbb{C})$, it means that $q$ is in $\mathcal{P}_{n}(\mathbb{C})$. Consequently, $z$ belongs to $Z_{\varepsilon}(p)$.

We are interested to know whether the real and the complex pseudozero sets are similar or not.

Let us first comment the following example considering $p(z)=1 / 2+z^{2}$. Figure 1 represents the real and complex pseudozero sets for this polynomial $p$ with $\varepsilon=0.1$. The real pseudozero set (Fig. 1b) is clearly enclosed in the complex pseudozero set (Fig. 1a). The shapes of the real and the complex pseudozero sets are quite similar. This example clearly exhibits that the real and the complex pseudozero sets look very similar

Let us now consider polynomial $q(z)=z-1$ with $\varepsilon=0.1$. Figure 2 again illustrates that the real pseudozero set (Fig. 2b) is included in the complex pseudozero set (Fig. 2a). Nevertheless, the real pseudozero set and the complex pseudozero set now have very different shapes. In fact, the real pseudozero set should be included in the real axis. Since it appears to not be the case here, we explain this phenomenon thanks to the following lemma.

Lemma 4.2 (Hinrichsen and Kelb [5]). The function

$$
d: \mathbb{R}^{n+1} \times \mathbb{R}^{n+1} \rightarrow \mathbb{R}_{+}, \quad(x, y) \mapsto d(x, \mathbb{R} y)
$$

is continuous at all pairs $(x, y)$ with $y \neq 0$ or $x=0$, and discontinuous at all pairs $(x, 0) \in \mathbb{R}^{n+1} \times \mathbb{R}^{n+1}, x \neq 0$.

This lemma states that a discontinuity problem arises when vector $y$ vanishes. In our case the discontinuity arises when $G_{I}(z)=0$, where $G_{I}$ is the imaginary part $G_{I}(z)$ of

$$
G(z)=\frac{1}{p(z)}\left(1, z, \ldots, z^{n}\right)^{T}
$$

It follows that $G_{I}$ vanishes for $z \in \mathbb{R}$, that is along the real axis. This explains why the contour function of MATLAB may fail and gives some bad results along the real axis. Of course, if none of the zeros of the polynomial is real, the real pseudozero set is reliable because we do not evaluate the function $G$ on the real axis. The first presented example and Figure 1 illustrate this case for instance. 


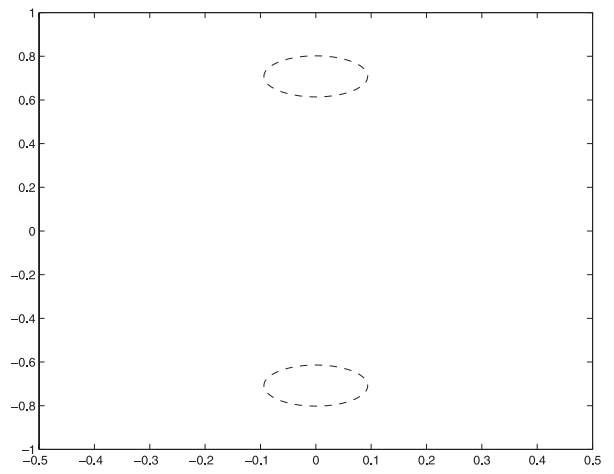

(a) Complex $Z_{\varepsilon}(p)$ with $\varepsilon=0.1$

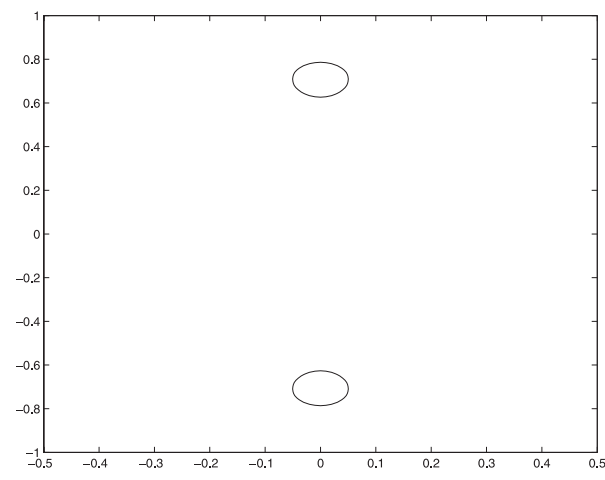

(b) Real $Z_{\varepsilon}^{R}(p)$ with $\varepsilon=0.1$

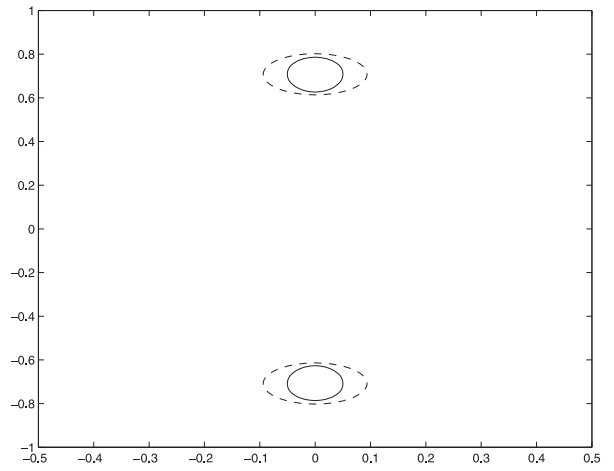

(c) Both sets on the same figure

Figure 1. Complex and real pseudozero sets for $p(z)=1 / 2+z^{2}$.

A reliable computation of the real pseudozero set on the real axis can be performed with the complex pseudozero counterpart thanks to the following lemma.

Lemma 4.3. Being $z \in \mathbb{R}, z$ belongs to $Z_{\varepsilon}^{R}(p)$ if and only if $z$ belongs to $Z_{\varepsilon}(p)$ (complex version of the pseudozero set).

Proof. This is true because the formula involved in Theorem 2.1 and in the proof stays in the real field if $z$ is real.

We conclude this comparison stating that in general complex pseudozero sets have to be preferred to real ones even to represent polynomials with perturbed real coefficients. Nevertheless real pseudozero sets yield an accurate and reliable description of perturbed zeros when it can be proved that none of them is real.

\section{ApplicAtions in CONTROL THEORY}

In control theory, classical transfer functions associated with some systems are often written as $H(z)=N(z) / D(z)$, where $N$ and $D$ are complex polynomials and 


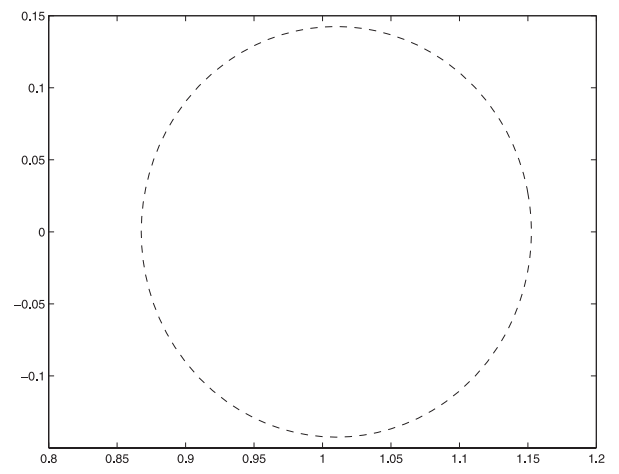

(a) Complex $Z_{\varepsilon}(q)$ with $\varepsilon=0.1$

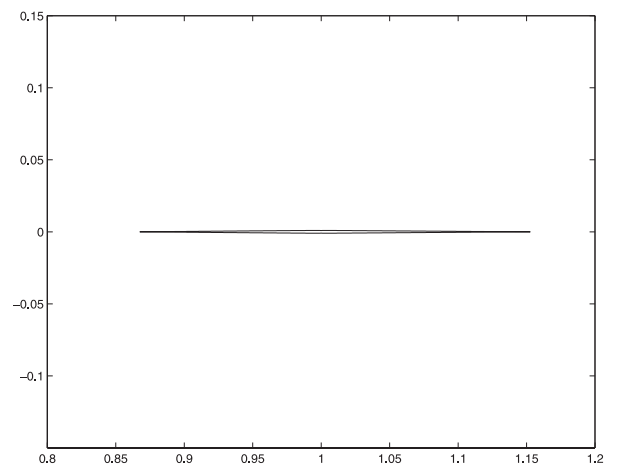

(b) Real $Z_{\varepsilon}^{R}(q)$ with $\varepsilon=0.1$

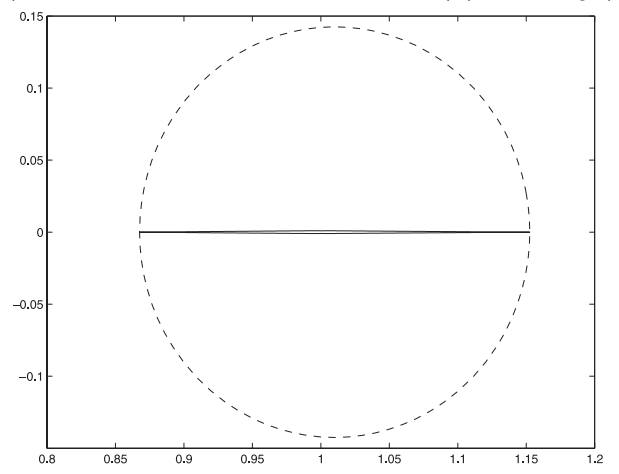

(c) Both sets on the same figure

Figure 2. Complex and real pseudozero sets for $q(z)=z-1$.

$z$ is a parameter of the system. The system described with the function $H$ is stable (in a sense to be defined later) if the polynomial $D$ is stable. Since uncertainties of the coefficients of the polynomials are unavoidable in most real problems (data uncertainty, rounding error), it is useful to know whether the system still remains stable while the coefficient uncertainty is bounded by a given $\varepsilon>0$. In such cases, the system is said to be robustly stable. Similarly the polynomial $D$ is robustly stable when it remains stable when its coefficients suffer from an uncertainty of $\varepsilon>0$. The robust stability of polynomial has been studied intensively (see [8] and the references therein) for polynomials with complex coefficients and with complex perturbations of those coefficients. Hereafter, we focus on stability for real polynomials only allowing real perturbations of the coefficients. We name this as the real stability problem.

\subsection{DeCiding ON POLYNOMIAL STABILITY: A FIRST CRITERION}

The real $\varepsilon$-pseudozero set can be used to decide the real stability of systems described by polynomials. A classic stability criterion in control theory is to compare 

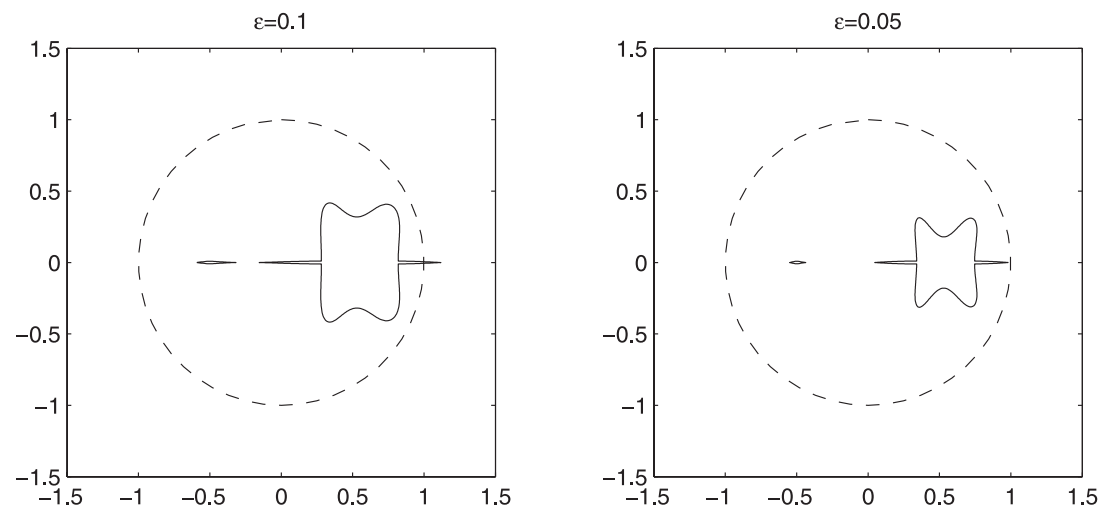

FiguRE 3. Real $\varepsilon$-pseudozero set of the polynomial $p(z)=(z-$ $0.3)(z-0.6)(z-0.7)(z+0.5)$, with $\varepsilon=0.1$ and $\varepsilon=0.05$.

the modulus roots to 1 (Schur stability). For a given polynomial with coefficients known with a tolerance $\varepsilon$, it is difficult to compute every root of all polynomials being in its $\varepsilon$-neighborhood. Of course, a sensitivity analysis that uses the condition number of the polynomial with respect to its coefficients can be performed and yields a first order criterion for arbitrary small uncertainty $\varepsilon$. Pseudozero sets provide a more general answer to this question since it neither neglect higher order effects of coefficient perturbations nor restrict these perturbations only to arbitrary small values. It suffices to draw the real $\varepsilon$-pseudozero set and to verify if it is included in the unit circle.

Figure 3 shows the real $\varepsilon$-pseudozero set of polynomial $p(z)=(z-0.3)(z-$ $0.6)(z-0.7)(z+0.5)$, with two coefficient uncertainties bounded by $\varepsilon=0.1$ and $\varepsilon=0.05$.

From this figure, we cannot decide the real stability for $\varepsilon=0.1$ because some 0.1-pseudozeros have modulus larger than 1. On the other hand, the figure proves that all the real 0.05-pseudozeros have a modulus less than 1 and so we conclude for the real stability of $p$ while $\varepsilon \leq 0.05$.

\subsection{DECIDING ON POLYNOMIAL STABILITY: A SECOND CRITERION}

Another kind of real stability is defined when the real part of all the roots of a polynomial are negative (Hurwitz stability). For testing this other real stability criterion, it suffices to draw the real $\varepsilon$-pseudozero set and to verify if it is included in the left half-plane. This is shown with Figure 4. We exhibit for example that polynomial $p(z)=(z+1)^{2}(z+0.2+0.2 i)(z+0.2-0.2 i)$ is real stable while $\varepsilon \leq 0.06$ but is unstable if $\varepsilon=0.6$. 

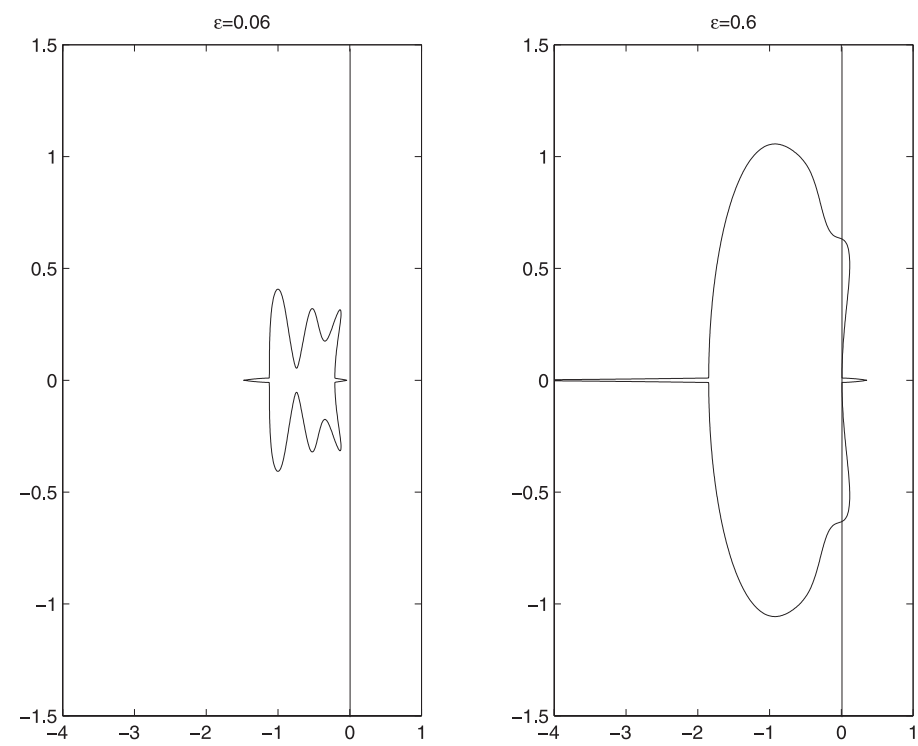

FiguRE 4. Real $\varepsilon$-pseudozero set of $p(z)=(z+1)^{2}(z+0.2+$ $0.2 i)(z+0.2-0.2 i)$, with $\varepsilon=0.06$ and $\varepsilon=0.6$.

\section{Conclusion}

In this paper, we have proposed a computable formula for the real pseudozero set. Identifying this set is motivated from data errors and rounding errors that corrupt the coefficients of real polynomials. Alas this formula is more difficult to compute than the corresponding complex one. Thanks to numerical simulations, we have shown that computing real pseudozero set may yield unreliable results. We justify this breakdown comes from the discontinuity of the level function. Then it is in general better to prefer the complex pseudozero set for real polynomial instead of the real pseudozero set.

Nevertheless, if the perturbations are sufficiently small to guarantee the zeros of the real polynomial stay far from the real axis then we may prefer to draw the real pseudozero set (since the discontinuity only appears near the real axis). In this case the real pseudozero set represents more accurately than before all the roots of the really perturbed polynomials.

\section{REFERENCES}

[1] J.-M. Chesneaux, S. Guilain and J. Vignes, La bibliothèque CADNA : présentation et utilisation. Manual, Laboratoire d'Informatique de Paris 6, Université P. et M. Curie, Paris, France, November 1996. Available at http://www-anp.lip6.fr/cadna/, (in French).

[2] W. Gautschi, On the condition of algebraic equations. Numer. Math. 21 (1973) 405-424. 
[3] S. Graillat and P. Langlois, Testing polynomial primality with pseudozeros, in RNC-5, Real Numbers and Computer Conference, Lyon, France, edited by M. Daumas (September 2003) 121-137.

[4] S. Graillat and P. Langlois, Pseudozero set decides on polynomial stability, in Proceedings of the Symposium on Mathematical Theory of Networks and Systems, Leuven, Belgium, edited by B. de Moor, B. Motmans, J. Willems, P. Van Dooren and V. Blondel (July 2004) (CD-ROM, papers/537.pdf).

[5] D. Hinrichsen and B. Kelb, Spectral value sets: a graphical tool for robustness analysis. Systems Control Lett. 21 (1993) 127-136.

[6] D. Hinrichsen and A.J. Pritchard, Robustness measures for linear systems with application to stability radii of Hurwitz and Schur polynomials. Internat. J. Control 55 (1992) 809-844.

[7] $W W W$ resources about Interval Arithmetic. http://www.cs.utep.edu/interval-comp/ main.html.

[8] L. Jaulin, M. Kieffer, O. Didrit and É. Walter, Applied interval analysis. Springer-Verlag London Ltd., London (2001).

[9] D.G. Luenberger, Optimization by vector space methods. John Wiley \& Sons Inc., New York (1969).

[10] R.G. Mosier, Root neighborhoods of a polynomial. Math. Comp. 47 (1986) 265-273.

[11] A.M. Ostrowski, Solution of equations and systems of equations. Second edition. Academic Press, New York. Pure Appl. Math. 9 (1966).

[12] H.J. Stetter, Polynomials with coefficients of limited accuracy, in Computer algebra in scientific computing - CASC'99 (Munich), Springer, Berlin (1999) 409-430.

[13] H.J. Stetter, Numerical Polynomial Algebra. Society for Industrial and Applied Mathematics (SIAM), Philadelphia, PA (2004).

[14] K.-C. Toh and L.N. Trefethen, Pseudozeros of polynomials and pseudospectra of companion matrices. Numer. Math. 68 (1994) 403-425.

[15] J. Vignes, A stochastic arithmetic for reliable scientific computation. Math. Comp. Sim. 35 (1993) 233-261.

[16] J.H. Wilkinson, Rounding errors in algebraic processes. Dover Publications Inc., New York (1994). 